\title{
Die Distanzierung von „Krawallmädchen“
}

\author{
Zur Rolle der Sprachwissenschaft in Debatten zur \\ gendergerechten Sprache
}

Zusammenfassung: In diesem Beitrag werden zwei österreichische Onlineforen zur gendergerechten Sprache auf sprachliches Wissen, die Verwendung von Fachvokabular und die Rolle der Sprachwissenschaft untersucht. Dabei wird die Diskussion mit der Fachdiskussion verglichen und Gemeinsamkeiten und Unterschiede werden herausgearbeitet.

Abstract: In this text two Austrian online discussion boards on gender-inclusive language are being analysed with regard to linguistic knowledge, the use of specialist vocabulary and the role of linguistics. The findings are compared to the academic discourse and their differences are discussed.

Schlüsselwörter: Geschlechtergerechte Sprache, Diskurs, Genderlinguistik

\section{Fragestellung und theoretische Grundlagen}

In der vorliegenden Untersuchung wird der Frage nachgegangen, welche Rolle sprachwissenschaftliches Wissen und die Sprachwissenschaft als Disziplin in Diskussionen zur geschlechtergerechten Sprache spielen. Dafür werden zwei Onlineforen untersucht, die zu Beiträgen der österreichischen Zeitung Der Standard eingerichtet wurden. Der erste Beitrag ist ein Aufruf des Vereins Deutsche Sprache, in dem die Abschaffung von geschlechtergerechter Sprache gefordert wird. Der zweite Beitrag ist die Replik einer Linguistin darauf.

Diese Analyse ist ein Beitrag zur Untersuchung des Diskurses zur gendergerechten Sprache in Österreich. Als Diskurs kann nach Gardt (2007: 30) eine Auseinandersetzung verstanden werden, die von unterschiedlichen gesellschaftlichen Gruppen mithilfe unterschiedlicher Äußerungen und Texte zu einem Thema geführt wird und das Wissen zu diesem Thema sowohl spiegelt als auch prägt. In dieser Untersuchung liegt der Fokus vor allem darauf, in wel-

Alker-Windbichler, Hannah: Universität Wien, Universitätsring 1, 1010 Wien, hannah.alkerwindbichler@univie.ac.at 
cher Form und von wem die Sprachwissenschaft in diese Auseinandersetzung eingebracht wird und welches sprachwissenschaftliche Wissen sich in dieser Diskussion findet.

Da es sich aber um ein begrenztes Korpus handelt, erhebt diese Untersuchung nicht den Anspruch, den Diskurs zur geschlechtergerechten Sprache in Österreich abzubilden. Vielmehr soll ein interessantes Diskursfragment in Bezug auf das Verhältnis von Sprachwissenschaft und Öffentlichkeit, Lai`innen und Expert*innen untersucht werden. Umgelegt auf die diskurslinguistische Mehrebenen-Analyse (DIMEAN) von Spitzmüller \& Warnke (2011), die drei Ebenen der Analyse vorsieht, liegt der Fokus hier aufgrund der begrenzten Datenmenge vor allem auf der intratextuellen Ebene und der Ebene der Akteure. Die transtextuelle Ebene zu erforschen, bleibt ein Forschungsdesiderat und erfordert ein größeres Korpus.

Auf der intratextuellen Ebene wird in der vorliegenden Diskussion vor allem die Zurschaustellung sprachlichen Wissens untersucht. Dabei soll ein besonderes Augenmerk darauf gelegt werden, ob linguistisches Fachvokabular verwendet wird oder die Ideen alltagssprachlich umschrieben werden. Weiters soll untersucht werden, ob diese Beiträge als Fakten präsentiert werden oder ob auf linguistische Autoritäten, sowohl Personen als auch Publikationen oder Institutionen, verwiesen wird. Die Analyse der Argumentation beschränkt sich also auf Argumente aus der Autorität, wobei es hier wesentlich ist, wie Bayer (2007: 133) ausführt, ob die angeführten Autoritäten tatsächlich auf dieses Gebiet spezialisiert sind. Ob und wie diese Autoritäten in den Diskurs eingebunden werden bzw. selbst darin auftreten, schlägt die Brücke zur Ebene der Akteure. Auch die Verwendung von Fachvokabular kann auf dieser Ebene als Differenzierung zwischen den Diskursteilnehmenden unter dem Aspekt des Vertikalitätsstatus (vgl. Spitzmüller \& Warnke 2011: 178) betrachtet werden.

Das Verhältnis der Postenden und anderer Akteure soll mithilfe sozialer Positionierung erfasst werden, denn der Diskurs zur geschlechtergerechten Sprache ist ein Metasprachdiskurs (oder sprachreflexiver Diskurs) und durch Sprachideologien, die in sprachreflexiven Diskursen zum Ausdruck kommen, ist es möglich, sich über Sprache sozial zu positionieren (vgl. Busch 2019: 132; Spitzmüller 2019: 27; Spitzmüller, Flubacher \& Bendl 2017: 8). Als Grundlage für die Beschreibung der Positionierung wird das Stance-Dreieck von Du Bois (2007) herangezogen. Dabei positionieren sich Akteure über die Bewertung eines Objekts zu diesem Objekt. Gleichzeitig richten sie sich über diese Bewertung auch zueinander aus. Das bewertete Objekt kann ein spezifischer Sprachgebrauch sein, z. B. die geschlechtergerechte Sprache. Dieser Vorgang ist ein sprachlicher Akt, der zugleich ein sozialer Akt ist (vgl. Du Bois 2007: 141). In der 
Erweiterung des Dreiecks durch Spitzmüller (2013: 270-273) richten sich Akteure über die Verknüpfung des Sprachgebrauchs mit einem sozialen Register nicht nur $\mathrm{zu}$ anderen Akteuren, sondern auch zu sozialen Personentypen aus und positionieren sich zu typisierten Verhaltensformen, da Sprachgebräuche im Kontext von Registern mit Personen- und Handlungstypen verbunden sind. In den hier untersuchten anonymen Diskussionen ist $\mathrm{zu}$ erwarten, dass sich die Positionierung hauptsächlich über Personen- und Handlungstypen gestaltet, da die Akteure sich nicht kennen und sich über die kurzen Kommentare nur bedingt aneinander ausrichten können. Daher wird mit Du Bois' einfachem Dreieck gearbeitet, wobei die Positionen entweder von zwei Akteuren oder einem Akteur in Ausrichtung zu einem Personentypus belegt werden können.

Bestrebungen, Sprache geschlechtergerecht zu gestalten, kamen aus der feministischen Linguistik (vgl. Pusch 1979; Trömel-Plötz 1978) und haben nicht zuletzt über zahlreiche Leitfäden Eingang in den offiziellen Sprachgebrauch gefunden (vgl. Wetschanow 2017). Dementsprechend wird die Verwendung gendergerechter Sprache mit einem bestimmten Personentypus (u. a. feministisch, politisch) assoziiert. Geschlechtergerechte Sprache ist über diese Zuschreibung mit dem Diskurs der Political Correctness verbunden und teilt sich mit ihm das Feindbild gesellschaftlicher Gruppen, die den öffentlichen oder persönlichen Sprachgebrauch aus ethischen Gründen regulieren möchten. In den hier untersuchten Kommentaren wird geschlechtergerechte Sprache auch mehrfach explizit der Political Correctness zugeordnet. Sie wird damit zu einer Frage der Moral (vgl. Stefanowitsch 2018) und zur Sprachpolitik im Sinne von policy (vgl. Teireck 2019: 383-384).

\section{Datengrundlage und methodisches Vorgehen}

Die Website der Zeitung Der Standard ist mit über 25000 Postings pro Tag (vgl. Huster 2018) eine der aktivsten Online-Plattformen im deutschsprachigen Raum und in Österreich für ihre sehr aktiven Userforen bekannt. Die zwei ausgewählten Beiträge sind keine redaktionellen Inhalte, sondern externe Kommentare, die in kurzer Abfolge im selben Medium erschienen, intertextuell verbunden und so gut vergleichbar in Hinblick auf die Rolle der Akteure sind, die in diesem Diskurs in Erscheinung treten.

Der erste Beitrag trägt den Titel Schluss mit dem Gender-Unfug! Ein Aufruf zum Widerstand aus dem Verein Deutsche Sprache und ist ein Appell des Vereins Deutsche Sprache zur Abschaffung der gendergerechten Sprache. Der Verein ist bei der für externe Kommentare üblichen Kurzinformation nicht näher be- 
schrieben, es finden sich in der Online-Ausgabe Berufs- bzw. Funktionsbezeichnungen wie „Schriftstellerin“ oder „Vorsitzender des Vereins Deutsche Sprache“ zu den Unterzeichnenden, in der Printausgabe nur ein Link zur Vereinswebsite. Unter den vier Unterzeichnenden gibt es eine Frau, die die Liste anführt. Der Artikel wurde online am 7. März 2019 publiziert und erschien in der Printausgabe am Weltfrauentag. Zum Zeitpunkt der Auswertung gab es 1405 Kommentare zum Artikel.

Die Replik darauf wurde von der Linguistin Claudia Posch verfasst und erschien am 21. März 2019. Sie wird sowohl online als auch in der Printausgabe als Assistenzprofessorin, Expertin für Feministische Linguistik und Mitglied im Verband für Angewandte Linguistik (verbal) vorgestellt. Zum Zeitpunkt der Auswertung gab es 752 Kommentare zum Artikel.

Die Threadstruktur macht es möglich, auf vorhandene Kommentare zu antworten. Von den insgesamt 2157 untersuchten Kommentaren sind 572 Kommentare der ersten Ebene, alle anderen sind bereits Antworten. Der längste Thread besteht aus 125 Kommentaren auf 19 Ebenen. Es sind also zwei Foren mit hoher Interaktion zwischen den Postenden. Die Zahl der an der Diskussion teilnehmenden Accounts kann nicht mehr genau ermittelt werden, da einige Accounts inzwischen gelöscht wurden und alle gelöschten Accounts gleich gekennzeichnet werden. Es kann von ungefähr 730 unterschiedlichen Accounts ausgegangen werden, wovon ca. 70 in beiden Foren aktiv waren.

Die Artikel und Kommentare können über die Website der Zeitung Der Standard abgerufen werden. Die Kommentare wurden als reiner Text erfasst und in einer QDA-Software mit Metadaten versehen. Die Metadaten enthalten eine fortlaufende ID, den Namen (bzw. Nickname) der postenden Person, den Zeitpunkt der Veröffentlichung, die Bewertung - jedes Posting kann von den Mitpostenden rot oder grün bewertet werden - und Angaben dazu, ob es ein Posting der ersten Ebene ist oder bereits eine Antwort auf ein Posting.

In der Software wurden induktiv Codes erstellt, um das Material $\mathrm{zu}$ erfassen. Die Codes wurden auf verschiedenen Ebenen vergeben, d. h. es wurden u. a. Codes für Themen, Akteure und Argumentation unterschieden. Für diese Untersuchung werden nun die Themen Linguistik, Wissenschaft und Universität und die Akteure, die aus dem Bereich der Sprachwissenschaft stammen, herangezogen. Diese Codes wurden bei knapp einem Viertel der Postings (insgesamt 504) vergeben. Dieser Wert ist bei beiden Foren gleich.

Die ID besteht aus „VDS-“ bzw. „P-“ (für Postings zum Artikel vom Verein Deutsche Sprache bzw. von Claudia Posch) und einer fortlaufenden Nummer und wird in diesem Text für Zitate verwendet, um die Postings zu anonymisieren. Der Reihung auf der Website folgend, die immer die aktuellsten Postings 
zuerst anzeigt, zeigt eine niedrige Nummer ein später verfasstes, eine hohe Nummer ein früher verfasstes Posting an. Sollten zitierte Postings von derselben Person (bzw. demselben Account) verfasst sein, wird gesondert darauf hingewiesen. Die Postings entsprechen nicht der schriftsprachlichen StandardOrthografie. Um den Lesefluss nicht durch mehrfache Kennzeichnungen von Orthografie-Abweichungen in jedem Zitat zu stören, werden fehlende Satzzeichen und abweichende Groß- und Kleinschreibung nicht mit „[sic]“ gekennzeichnet.

\section{Ergebnisse der Analyse}

Eine Analyse von 2157 Kommentaren lässt sich aus zahlreichen Blickwinkeln durchführen. Die zwei Foren sind ein reichhaltiger Datenschatz, nicht nur in Bezug darauf, was über geschlechtergerechte Sprache als sprachliche Form gesagt wird, sondern auch in Bezug darauf, welche Themen damit verknüpft werden. Besonders häufig wird der gefühlte Zwang, diese Sprachformen zu verwenden, diskutiert, auch in Zusammenhang mit bestimmten Institutionen und der von ihnen ausgeübten Macht.

Aber auch Themen, die sich von der geschlechtergerechten Sprache entfernen, wurden bei der Auswertung der Foren erfasst und gesammelt. Sie reichen von naheliegenden Diskussionen, wie zur mangelhaften Rechtschreibung und Grammatik in konkreten Postings, durch deren Thematisierung die Berechtigung, sich zu diesem Thema zu äußern, strittig gemacht wird, bis hin zu weniger naheliegenden Themen, wie der korrekten Pluralbildung von Semmelknödeln, wenn sie aus einer oder mehreren Semmeln bestehen, und persönlichen Schulerfahrungen.

Um dem Gegenstand des Sammelbands möglichst nahe zu bleiben, beschränkt sich die Analyse auf drei Themenbereiche. Als erstes soll die Bezugnahme auf die Linguistik als Autorität bzw. die Bezugnahme auf konkrete Personen aus dieser Disziplin untersucht werden. Da es sich gezeigt hat, dass hier auch die Germanistik als Expertendisziplin in Erscheinung tritt, werden beide Disziplinen zusammengenommen. Danach bewegt sich der Fokus von der Wissenschaft als Abstraktum zu dem konkreten Ort der Wissenschaft in der Gesellschaft, der Universität. Anschließend soll die Verwendung von Fachvokabular und Darstellung von Fachwissen untersucht werden. 


\subsection{Linguistik und Germanistik}

Die zwei Foren unterscheiden sich in der Dichte der Verweise auf Germanistik und Sprachwissenschaft. Das lässt sich unter anderem auf die unterschiedlichen Ausgangstexte zurückführen. Im ersten Artikel wird die Sprachwissenschaft nicht erwähnt und auch kaum Fachvokabular verwendet. Lediglich in Bezug auf den „Generalirrtum“ werden die Begriffe „natürliches“ und „grammatisches Geschlecht“ (Maron et al. 2019: o. S.) verwendet. Auch in den Informationen zu den Autor*innen werden diese nicht als Germanistinnen oder Linguist`innen bezeichnet. Anders ist das beim zweiten Text, an dessen Ende Claudia Posch als „Assistenzprofessorin im Bereich Sprachwissenschaft an der Universität Innsbruck, Expertin für Feministische Linguistik und Mitglied im Verband für angewandte Linguistik Verbal“ (Posch 2019: o. S.) bezeichnet wird. In dem Text selbst wird auch auf zwei linguistische Veröffentlichungen, nämlich Kalverkämper (1979) und Pusch (1979), verwiesen und ausdrücklich eine „linguistische Perspektive“ (Posch 2019: o. S.) eingenommen. Es finden sich auch Fachausdrücke, wie „generisches Maskulinum“ oder „Mehrworteinheiten“ (Posch 2019: o. S.), die jedoch im Text erklärt werden.

Die unterschiedlichen Ausgangstexte spiegeln sich in der Lexik der Kommentare wider. So findet sich der Begriff „Linguistik“ 17-mal im Forum zu Claudia Posch, und ist dabei in 15 Fällen eine Reaktion auf ihre Kurzbiografie. Im VDS-Forum kommt der Begriff nur zweimal vor, wobei er sich auch dort einmal auf Claudia Posch bezieht. Der Begriff „Sprachwissenschaft“ bzw. „Sprachwissenschaften" ist auch bei dem Text der Sprachwissenschaftlerin in Proportion zur Gesamtmenge der Postings häufiger in den Reaktionen enthalten. Es zeigt sich also schon an einer kurzen quantitativen Aufstellung wenig überraschend, dass die Linguistik stärker thematisiert wird, wenn ihre Vertreter`innen aktiv in die Diskussion eintreten.

Die Referenz auf die Kurzbiografie wird meist dazu genutzt, Linguistik und Feminismus als unvereinbar zu zeichnen. Das kann explizit passieren, wie z. B. in diesem Kommentar:

Mir stellen sich erst recht die Haare auf bei dem Begriff „feministische Linguistik“. Der zeigt nämlich, daß hier zwei wesentliche Kriterien für Wissenschaftlichkeit verletzt sind: Die Ergebnisoffenheit und die Tatsache, daß Wissenschaft nur beschreiben aber niemals werten darf! [...]

Die Wertung kann aber auch angedeutet bleiben, z. B. „,Expertin für Feministische Linguistik، Kann man nicht erfinden.“ (P-483). Der Vorwurf der Pseudowissenschaft wird in den Kommentaren nicht nur gegen die feministische Lin- 
guistik, sondern auch gegen Gender Studies erhoben, die in den Ausgangstexten nicht erwähnt werden. In manchen Fällen wird die Möglichkeit von kulturwissenschaftlicher Erkenntnis insgesamt infrage gestellt: „Wer objektiv recht hat? Wird nie festgestellt werden können. Weil es subjektiv ist.“ (P-032)

Häufig wird ein Punkt aufgegriffen, der nur in einem der Artikel angedeutet wird, nämlich die Frage von empirischen Studien. Posch (2019: o. S.) schreibt, dass „[a]lle dahingehenden empirischen Belege von angesehenen Expert^innen“ in der Diskussion ignoriert werden, führt aber keine Studien an. Manche Postende meinen aber zu wissen, um welche Studien es sich handelt:

Die Studien, die Posch erwähnt, haben oft meth. Schwächen. So wurde in einer Studie gefragt, ob Mädchen sich eher zutrauen würden Astronaut oder Blumenverkäuferin zu werden. Als dann die meisten gesagt haben „Blumenverkäuferin“, wurde daraus messerscharf geschlossen, dass der Grund nicht die Schwierigkeit der Berufe sei, sondern sprachliche Ursachen habe.

Es wird zum Teil auch die Existenz dieser Studien verneint, z. B. „Nein, solche Studien gibt es nicht! Und wenn in Aufsätzen von Neusprech-Befürwortern Studien erwähnt werden, dann meistens grob verzerrt.“ (P-286). Oder die Möglichkeit, kognitive Abbilder überhaupt empirisch zu erheben, wird in Zweifel gezogen:

Bei dem Hinweis auf die Belege bez. der kognitiven Verarbeitung der „ausschließlich maskulinen Form“ bin ich etwas skeptisch. Das Problem bei der Erhebung ist, dass man dabei auf introspektive Verfahren angewiesen ist, die allein schon durch die Fragestellung das Ergebnis beeinflussen. [...]

$(\mathrm{P}-281)^{1}$

Manche Postende suchen frei verfügbare empirische Studien im Internet und verlinken diese, häufiger wird jedoch auf Zeitungsartikel, Blogs und YoutubeVideos verwiesen. Es wird also von einer kleinen Gruppe durchaus versucht, den aktuellen Stand der empirischen Forschung zu diskutieren, wenn auch ohne Unterscheidung zwischen Soziologie, Politikwissenschaft und Linguistik, es fehlt dazu aber der Zugang. Wie ein Kommentar nach einem geposteten Link zu einem Artikel (Pérez \& Tavits 2019) festhält: „Ist leider hinter einer Paywall“ (VDS-0386). Nur indirekt wird auf linguistische Studien in der Diskussion ver-

1 An dieser Stelle soll angemerkt sein, dass Introspektion nicht das Verfahren ist, mit dem in kognitions- und psycholinguistischen Untersuchungen kognitive Abbilder erhoben werden (vgl. Gygax et al. 2008, Kusterle 2011, für eine Übersicht Kotthoff \& Nübling 2018: 91-127). 
wiesen, durch einen Link zu dem Blog Belles Lettres von Daniel Scholten, der sich in einem längeren Video mit empirischer Forschung der feministischen Linguistik auseinandersetzt (vgl. Scholten 2018). Die Studien selbst und ihre Ergebnisse kommen in der Diskussion nicht vor.

Bei den Referenzen auf Personen soll hier zwischen generischen, nichtspezifischen und spezifischen Referenzen unterschieden werden (vgl. Pettersson 2011: 63), auch wenn diese Unterscheidung bei Kommentaren mit wenig Kontext nicht immer leicht $\mathrm{zu}$ treffen ist. Spezifische Referenzen auf Linguistinnen und Germanistinnen gibt es abseits der Beschäftigung mit der Kurzbiographie in Referenzen auf Luise F. Pusch, die bereits im Text von Posch erwähnt wird, auf eine nicht näher beschriebene „Linguistin auf der Uni“, die „das Gendern als unzulässige Bedeutungsverschiebung“ beschrieben habe (VDS-0486) und durch das Verlinken eines Youtube-Videos einer Rede von Tomas Kubelik, einem Deutschlehrer und Autor des Buchs Genug Gegendert! (VDS-0578-VDS-0582).

Es gibt wenige Selbstbezeichnungen, wie „Ich bin Deutschlehrer“ (VDS0531), „als Germanist“ (P-215), „als Sprachwissenschafterin“ (VDS-0937) und die Bezeichnung von Posch als „werte Kollegin aus Innsbruck“ (P-222), die einen fachlichen Hintergrund nahelegt. Dabei zeigt sich, dass ein frühes Outing als Fachperson die Interaktion mit anderen Postenden hemmt und wenig Reaktionen auslöst. Das könnte mit der Idee des Forums als Austauschort Gleichberechtigter zu tun haben, wie es an einer Stelle im Rahmen einer Diskussion über korrekte Orthografie explizit gemacht wird: „das ist hier keine Universitätsvorlesung, sondern ein öffentlicher Diskussionsraum“ (P-584). Zu diesem egalitären Raum steht die Wahrnehmung von Vertreter^innen geschlechtergerechter Sprache als elitär im klaren Widerspruch.

Unter den nicht-spezifischen Referenzen ist ein Austausch besonders aufschlussreich, in dem Genderlinguistinnen zunächst als nicht „linksliberal“ bezeichnet werden, da sie Sprachentwicklung „,von oben“ diktieren wollen (P062), woraufhin eine „eindeutige Distanzierung von diesen Krawallmädchen“ (P-063) gefordert wird. Wer sich nun in wessen Namen von den Vertreterinnen der Genderlinguistik distanzieren soll, wird nicht weiter ausgeführt. Es ist eine von mehreren Stellen, an denen versucht wird, Genderlinguistik oder feministische Linguistik als abseits des wissenschaftlichen Mainstreams darzustellen. So wird sie beispielsweise als „Außenposition, die von einigen Soziolinguisten eingenommen wird“ (P-228) bezeichnet, ohne diese Behauptung weiter auszuführen oder zu belegen. Diese Strategie ist relevant in Hinsicht auf das Prestige, das Sprachwissenschaft als Referenz genießt. Der Ausdruck „Krawallmädchen“ zeigt auch an, dass in diesem Diskurs neben Geschlecht, Bildung und sozialer 
Zugehörigkeit auch das Alter bzw. der Generationenkonflikt eine wichtige Rolle spielt. Das Alter der Personen wird nicht öffentlich in den Foren angegeben, wird aber von den Postenden selbst als Konfliktthema angesprochen, z. B. „die Opa-Generation“ (P-192) oder „stirbt aber mit euch auch aus“ (VDS-1279). Hinweise auf das Alter gibt es auch durch den Verweis auf Universitätserfahrungen vor und nach Bologna oder durch die Verwendung reformierter oder nichtreformierter Rechtschreibung. Wie auch bei Selbstzuschreibungen eines Geschlechts kann die Selbstzuschreibung eines bestimmten Alters nicht verifiziert werden.

Generische Referenzen auf Germanist*innen und Linguist*innen zeugen im Gegensatz zu den spezifischen Referenzen von einem hohen Status und bleiben in der Diskussion unwidersprochen. Dieser Status mag dadurch begründet sein, dass eine „Germanistin oder ein[] Germanist[] deines Vertrauens“ (VDS-0081), die „Linguistikexperten“ (P-012) oder die „linguistikerInnen“ (P-512) immer der eigenen Meinung sind und zur Unterstützung derselben zitiert werden. Auch die Sprachwissenschaft oder die Germanistik als Disziplinen haben ein hohes Ansehen und treten ungeachtet der Auseinandersetzung um feministische Linguistik als monolithische Gebilde auf, die die Argumente der Postenden unterstützen, wie dieses Beispiel zeigt, bei dem diese Disziplinen als Antwort auf die Frage nach dem Ursprung einer aufgestellten Behauptung angeführt werden und weiter ausgeführt wird:

[Germanistik und Sprachwissenschaften] sind nämlich jene wissenschaftlichen Fachrichtungen, die da tatsächlich wissen, was Sache ist, anstatt aus ideologischer Verblendung die Realität mit Sophismen und emotionaler Erpressung wegerklären zu wollen, wenn sie einem nicht gefällt.

(VDS-0093)

Eine positive Auffassung von Geistes- und Kulturwissenschaften wird aber nicht grundsätzlich in den Postings vertreten, denn gleichzeitig wird auch häufig von den „richtigen Wissenschaften“ gesprochen, die sich dem „Trend“ geschlechtergerechter Sprache verweigern bzw. ausschließlich auf Englisch publizieren (VDS-1063/64). Die Sprachwissenschaft wird hierbei jedoch nicht explizit als Teil der Geistes- und Kulturwissenschaften genannt, sondern im Gegenteil anderen gegenübergestellt:

Und wo ist jetzt der „linguistische Beweis“, dass es sich beim Gendern nicht um „Unfug“ handelt? Alles was man hier lesen kann sind politische bzw soziologische „Argumente“, denen Werturteile zugrunde liegen, genauso wie der „Unfug“-Bewertung. 
Oder es wird in Bezug auf eine diskutierte Studie festgestellt: „Die Autorinnen sind auch Psychologinnen und haben keine Ahnung von Sprachwissenschaften.“ (P-293) Die Sprachwissenschaft und die Germanistik treten in diesem Diskursfragment grundsätzlich als validere, vertrauenswürdigere Wissenschaften auf als Gender Studies oder Soziologie. Gerade für die Gegner*innen der geschlechtergerechten Sprache sind sie meist die einzigen Wissenschaften, die hier als Expertendisziplinen infrage kommen, da der Einfluss von gesellschaftlichen Faktoren auf den Sprachgebrauch als unzulässiger Eingriff abgelehnt wird.

Anders als die Sprachwissenschaft ist das Expertentum nicht grundsätzlich positiv. Neben der Beschäftigung mit der betreffenden Stelle in der Kurzbiografie von Posch („Expertin für feministische Linguistik“s. o.), die mehrfach in den Kommentaren zitiert und kommentiert wird, gibt es vereinzelt auch den Aufruf, Expertisen grundsätzlich zu misstrauen: „Experten und -innen ,beweisen“ mal dies, mal das, je nachdem, wie man's braucht.“ (P-687) In diesem Posting werden alle Wissenschaften als „ideologisch instrumentalisierbar“ bezeichnet und ein Vergleich mit Glyphosat-Gutachten und Rechtsgutachten zugunsten von Immobilienkonzernen gezogen. Dieser Seitenstrang der Diskussion verweist auf die immer wieder geäußerte Vorstellung einer diskursbestimmenden, unaufrichtigen oder uninformierten Elite, die im Zusammenhang mit Universitäten als Bezugsort noch stärker in den Vordergrund tritt.

Die Linguistik und Germanistik sowie ihre generischen Vertreter`innen werden also in dieser Diskussion als Autoritäten angeführt. Sobald jedoch aus diesem vagen Verweis eine spezifische Referenz wird, wird diese Position in der Regel angegriffen. Dabei werden aber nicht die Sprachwissenschaft oder Germanistik durch eine Ganzes-Teil-Argumentation (vgl. Kienpointner 1992: 274284) beim Angriff auf ihre Vertreter`innen in Mitleidenschaft gezogen, sondern es folgt ein argumentum ad hominem und die Person wird als nichtrepräsentativ für diese Disziplinen bezeichnet.

\subsection{Universitäten als Bezugsort}

Die Hochschule als der Ort des vermeintlich verbindlichen Genderns wird in der österreichischen Öffentlichkeit seit Jahren immer wieder diskutiert. Angestoßen durch Medienberichte leitete die österreichische Volksanwaltschaft, ein Kontrollorgan der öffentlichen Verwaltung, 2015 eine Prüfung ein, ob die Verwendung einer geschlechtsneutralen Sprache an österreichischen Hochschulen als Beurteilungskriterium schriftlicher Arbeiten festgelegt sei und ob solche Vorgaben verfassungswidrig seien, da sie orthografisch nicht der deutschen Sprache 
entsprechen, die als „Staatssprache“ im Bundesverfassungsgesetz festgelegt ist. Sie konnte keine allgemeinen, verbindlichen Vorgaben an den Universitäten finden, jedoch an einigen Fachhochschulen und Pädagogischen Hochschulen (vgl. Volksanwaltschaft 2018). Der Ring Freiheitlicher Studenten (RFS), dessen Anliegen einen Punkt „Nein zum Genderwahn“ beinhaltet (RFS o. J.), initiierte 2017 eine Bürgerinitiative „Wissenschaftliche Arbeiten genderfrei!“ (RFS 2017). Das zuständige Ministerium sah das Benotungskriterium geschlechtergerechte Sprache aber sowohl durch das Bundes-Gleichbehandlungsgesetz als auch als Teil der universitären Autonomie gedeckt (BMWFW 2017).

Auch in den vorliegenden Daten ist dieser Konnex stark gegeben. Die Universität wird als einer der wenigen Orte gezeichnet, an dem das Gendern zum Alltag gehört, durch den Zwang, schriftliche Arbeiten gendergerecht zu gestalten:

Im Endeffekt ist das Gendern ohnehin nur was für den Elfenbeinturm der Universitäten und ein paar Juristen, die Gesetze/Verordnungen schreiben müssen. Überall sonst und schon gar nicht in der gesprochenen Sprache wird es sich zurecht nie durchsetzen und auf Ablehnung stoßen. Leid tun mir nur die Studenten, meist Frauen, die in Geistes- und Sozialwissenschaften damit gepflanzt werden, in ihren Abschlussarbeiten irgendwelche kruden Genderregeln zu befolgen.

(VDS-0734)

Das Argument des Zwangs ist zentral, da dies ein wichtiges Argument in der ursprünglichen Auseinandersetzung war. Posch argumentiert am Ende ihres Beitrags, dass der VDS durch den Appell „an Politiker, Behörden, Firmen, Gewerkschaften, Betriebsräte und Journalisten“, die deutsche Sprache „gegen diesen Gender-Unfug“ durchzusetzen (Maron et al. 2019: o. S.), selbst anderen vorschreiben möchte, wie diese „mit ihrer Sprache umgehen dürfen“ (Posch 2019: o. S.). Auch allen Seiten in der Diskussion in den Foren ist gemein, abzustreiten, selbst Macht auszuüben. Während die einen von „einer kleinen privilegierten pseudoelitistischen Minderheit angeordnete[n] Änderung der Sprache“ (VDS-0150), von einer „Zwangsänderung“ (VDS-0326), einem „durch und durch totalitäre[n], top down Ansatz“ unter dem „Sauerstoffzelt des permanenten staatlichen Zwangs“ (VDS-0562) sprechen, meinen andere: „Aber kein Mensch zwingt irgendjemanden das umzusetzen." (VDS-0575)

Tatsächlich scheint für viele die Anwendung von geschlechtergerechter Sprache ohne Zwang unvorstellbar, und es herrscht die Vorstellung, dass dieser Zwang vor allem aus Mechanismen des gesellschaftlichen Ausschlusses besteht, die mit der „Angst vor dem Hass einiger weniger“ (VDS-0334) und „oft und gerne mit manchmal mehr und manchmal weniger subtiler emotionaler Erpressung“ (VDS-0015) „bei Sanktion der gesellschaftlichen Ächtung“ (VDS- 
0150) funktionieren, denn „wer es nicht macht oder ablehnt, wird in manchen Kreisen gleich als Sexist dargestellt“ (VDS-1402). Hier wird immer wieder die Vorstellung einer mächtigen Minderheit bemüht, von „selbstgerechte[n] Wichtigtuerinnen, die mir eigentlich gar nichts zu sagen haben“ und die „glauben, der ganzen Gesellschaft Vorschriften machen zu können“ (VDS-0170). Da es an Hochschulen ein klar definiertes Machtgefälle gibt und die Kriterien für das Erreichen eines positiven Abschlusses einseitig definiert werden, werden sie als einer der Orte dieser mächtigen Minderheit gesehen. Denn die Universitäten sind „dort, wo sie [die ,Genderistinnen'] ihr kleines bisschen Macht haben“ (P234) und diese Macht wird ausgenutzt: „Die sind da kompromisslos und zwingen die Studenten zu gendern.“(VDS-1404)

An diesem Punkt konvergieren drei wichtige und wiederkehrende Topoi in den Daten: 1. Ideologie-Topos: Gendern beruht auf einer ideologischen Verblendung, nicht auf wissenschaftlichen Erkenntnissen, 2. der Topos des natürlichen Wandels: Sprachwandel darf nur natürlich und ohne Zwang passieren, 3. Mehrheitstopos: Was von der Mehrheit abgelehnt wird, darf nicht zur Norm erhoben werden. Diese drei grundlegenden Argumentationsmuster werden eng mit der politischen Landschaft in Österreich verknüpft: Das Gendern wird immer wieder mit rot-grün oder links in Verbindung gebracht, z. B.:

Der Aufruf der VDS ist ein Widerstand gegen die von rot-grünen Politikern, Genderwissenschaftler [sic] und Journalisten betriebene Änderung der Sprache die vollkommen ohne Beteiligung der Bevölkerungerfolgen [sic] soll.

Die Universitäten, insbesondere die Geisteswissenschaften, werden dabei als Ort der linken Indoktrinierung gezeichnet: „Die Geisteswissenschaften sind wirklich von radikale [sic] Linken, die offensichtlich eine Identitätspolitik betreiben, übernommen worden.“ (P-379) und die Verwendung von geschlechtergerechter Sprache dient, ähnlich wie in „kommunistischen Diktaturen“, dem „Haltung beweisen“ (VDS-0165).

Als Autorität für diese vermutete linke Unterwanderung der Geisteswissenschaften wird Jordan Peterson zitiert, ein kanadischer Professor für Psychologie mit einer großen Reichweite in den sozialen Medien, der bereits 2016 angekündigt hatte, lieber ins Gefängnis und in den Hungerstreik zu gehen, als sich vorschreiben zu lassen, mit welchen Pronomen er Menschen zu bezeichnen hätte, und der bei seinen Anhänger`innen als „Tabubrecher“ (vgl. Dietz 2018) gilt. Auf seinem Youtube-Kanal, der über 2,5 Millionen Abonnent`innen aufweist, gibt es eine Playlist mit dem Titel „Professor against Political Correctness“ (vgl. Peterson 2019). 
Durch den in den Foren immer wiederkehrenden Verweis auf die Vielfalt von Personalpronomen und Geschlechtern wird auch unabhängig von der Person Peterson eine Verbindung zu Universitäten in den USA und Kanada und zu dem Diskurs der Political Correctness aufgezeigt. So wird von einer Pflicht an einigen Universitäten „eigene Pronomen für die verschiedenen Geschlechter“ (VDS-0444) zu verwenden und von einem „Wildwuchs an frei erfundenen Personalpronomen für diverse selbst erfundene Geschlechter“ (VDS-0121) gesprochen. Das steht im Widerspruch zu der immer wieder aufgestellten Behauptung, geschlechtergerechte Sprache sei „hauptsächlich eh nur im deutschsprachigen Raum“ (P-155) ein Problem und das Englische habe es nicht nur leichter, sondern würde sogar eine „gegenläufige Tendenz“ (VDS-0786) aufweisen. Die Universität wird also auch international als ein Ort gesehen, der den Empfindungen der Mehrheit zuwiderläuft.

Eine Rolle spielen dabei auch eigene Universitätserfahrungen und die Zeit, die seit ihnen vergangen ist, denn wie bei den oben erwähnten „Krawallmädchen“ kommt auch hier wieder das persönliche Alter als Variable ins Spiel. Als prestigereicher Ort der Vergangenheit verleiht er den eigenen Argumenten Nachdruck. So argumentiert eine Person, sie habe „auf der Uni Arbeiten auf Englisch und Spanisch geschrieben, aber alle in einem grammatikalisch korrektem [sic] Englisch und Spanisch“, während heutzutage auf den Universitäten „GRAMMATIKALISCH FALSCHES Deutsch“ (VDS-0582) gefordert würde. Verweise auf die eigene Biografie sind in den Daten jedoch selten explizit, öfter wird durch Fachvokabular (vgl. Abschnitt 3.3) eine Verbindung zur Wissenschaft hergestellt und ohne konkreten Bezug auf die eigene Person der aktuelle Zustand der Hochschulen beklagt.

Ob universitäre Erfahrungen stattgefunden haben, kann in einem anonymen Online-Forum nicht überprüft werden. Ein Austausch beginnt mit einem Verweis auf die Publizistin Birgit Kelle, die in der deutschen Zeitschrift Merkur über die Verpflichtung zur geschlechtergerechten Sprache bei einer Arbeit über Prostata-Patienten spricht (vgl. Kelle \& Rohleder 2015), und führt zu einer Debatte zweier User*innen, wie in einer Dissertation zitiert wird und in weiterer Folge auch, wer sich zum wissenschaftlichen Schreiben äußern darf: „Da sie offenbar noch nie eine Doktorarbeit verfasst haben, ist es sinnfrei, mit ihnen weiter darüber zu debattieren.“ (P-213) Auf die Antwort „Ich habe schon viele wissenschaftliche Arbeiten verfasst. Das ist nämlich mein Beruf [...]“ (P-214) und die Einmischung eines weiteren Users, „OMG! sollte es Ihnen gelungen sein, eine Diss als reine Montage aus Zitaten zu verfassen? Da dürfen Sie natürlich nicht hinein gendern. Dieses Sprachkunstwerk würde mich als Germanist sehr interessieren. ;-)“ (P-215), endet dieser Thread ohne weitere Interaktion. Auch 
hier wird die eigene universitäre Erfahrung, also das Schreiben einer Dissertation, nicht explizit angeführt, stattdessen werden allgemeine Grundsätze über das Schreiben wissenschaftlicher Texte aufgestellt. An den Antworten ist aber ersichtlich, dass diese Äußerungen biografisch verstanden werden. Wie auch an anderen Stellen führt das Herstellen eines fachlichen Bezugs zum Abbruch der Kommunikation.

Es gibt vereinzelt auch Erfahrungsberichte aus den Hochschulen, die aus der Zeit nach dem Bologna-Prozess und daher vermutlich von jüngeren User^innen stammen, wie den Verweis auf eine Bachelor-Arbeit, die „in der ersten Abgabe aufgrund Verstoß gegen Genderrichtlinen [sic] negativ bewertet“ (VDS-1402) wurde oder auf eine anstehende Bachelor-Arbeit, die eine Userin „auf englisch verfassen oder [für die sie] aus Überzeugung eine schlechtere Note in Kauf nehmen“ (VDS-0164) möchte.

In der Diskussion nimmt die Universität also einen doppelten Platz ein, zum einen als vergangener, prestigereicher Ort, zum anderen als aktueller Ort, zu dem keine positive Beziehung hergestellt wird: Während formale Bildung immer wieder angedeutet wird, gibt es in den 2157 Kommentaren keine Äußerung, die explizit auf ein aktuelles Anstellungsverhältnis oder sonstiges Naheverhältnis zur Universität verweist.

Mehr als über Nutzen und wissenschaftliche Fundierung erfolgt hier die Abgrenzung von Gegner`innen dieser Sprachformen über die negative Bewertung eines vermeintlichen Zwangs. Nicht nur die geschlechtergerechte Sprache an sich dient also als Objekt zur Positionierung, sondern auch der Zwang kann als Objekt in ein Stance-Dreieck eingesetzt werden. Damit positionieren sich die Akteure demokratischer als (der Personentypus der) Feministinnen.

\subsection{Fachvokabular und sprachliches Wissen}

Wenn die „Genderisten“ (P-033) als Personentypus in einem Stance-Dreieck geschlechtergerechte Sprache als wissenschaftlich fundiert und als Mittel zur positiven gesellschaftlichen Veränderung sehen, müssen diejenigen, die diesen „Gender-Unfug“ (Maron et al. 2019) ablehnen, diesen Sprachgebrauch auch anders bewerten, um sich von dieser Gruppe abzugrenzen. Der positive gesellschaftliche Nutzen für Frauen ist tatsächlich ein großes Thema in der Debatte, auf das hier nicht näher eingegangen werden kann. An dieser Stelle soll es um die wissenschaftliche Fundierung gehen, die in den Kommentaren großteils in Abrede gestellt wird.

Die zentrale Frage in der Debatte zur geschlechtergerechten Sprache ist die Frage des Verhältnisses von Genus und Sexus. Sie steht nicht nur am Beginn 
der Auseinandersetzung in der germanistischen Linguistik Ende der 1970er Jahre (vgl. Trömel-Plötz 1978; Kalverkämper 1979; Pusch 1979), sondern beschäftigte bereits Protagoras und viele seit ihm (vgl. Irmen \& Steiger 2005: 214215). Im 19. Jahrhundert setzt sich die Sichtweise von Jacob Grimm, der im Gegensatz zu Karl Brugmann eine Verbindung zwischen der männlichen und weiblichen Natur und den Genera sah, weitgehend durch (vgl. Irmen \& Steiger 2005: 220-222; Leiss 1994: 289). Im 20. Jahrhundert etablierte sich jedoch, theoretisch fundiert durch Roman Jakobsons Markiertheitstheorie, das Konzept des generischen Maskulinums, das besagt, dass das Maskulinum geschlechtsabstrahierend zu verstehen ist (vgl. Doleschal 2002: 62).

Das generische Maskulinum wurde von der feministischen Linguistik stark kritisiert und inzwischen belegen zahlreiche empirische Untersuchungen, dass das Maskulinum nicht geschlechtsabstrahierend verstanden wird (vgl. Braun et al. 1998; Irmen \& Linner 2005; Gygax et al. 2008; Kusterle 2011), zumindest nicht in einer Weise, die Männer und Frauen gleichmäßig repräsentiert. Kotthoff \& Nübling (2018: 115) führen das unter anderem auf einen male bias zurück, der auch bei Neutralisierungsstrategien wirksam wird, und betonen die Vielfalt an Faktoren, die Geschlechterrepräsentanz beeinflussen.

Der Ausgangsartikel von Posch adressiert das Ungleichgewicht, das das generische Maskulinum hervorruft: „Das sogenannte generische Maskulinum führt außerdem dazu, dass sich Kinder eine in Schieflage geratene Welt vorstellen: eine Welt, in der männliche Menschen immer wichtiger und aktiver sind als alle anderen“ (Posch 2019: o. S.). Damit wird die aktuelle, empirische Forschung angesprochen. Wie oben erwähnt, gibt es in der Diskussion den Versuch, aktuelle Forschung zu diskutieren, was jedoch an der Verfügbarkeit aktueller linguistischer Ergebnisse scheitert. In den Kommentaren werden Studien auch explizit verlangt, und wenn sozialwissenschaftliche oder psychologische Studien in den Kommentaren verlinkt oder beschrieben werden, werden die Daten kritisch diskutiert.

Während die Frage der empirischen Forschung in einem der Ausgangstexte angesprochen wird, wird die Frage der Korrektheit von Sprachformen in keinem der Texte explizit behandelt. Der VDS spricht von „lächerlichen Sprachgebilde[n]“ und „Verzerrungen der Sprache“ (Maron et al. 2019: o. S.), worauf Posch kontert, dass Deutsch „eine außerordentlich flexible Sprache“ (Posch 2019: o. S.) sei. Im Gegensatz dazu ist die Frage nach Richtig und Falsch in den Kommentaren sehr ausgeprägt. Während sich die Frage nach der Korrektheit sprachlicher Formen in der Linguistik oft nicht stellt, ist es für viele NichtLinguist*innen selbstverständlich, dass bei mehreren Varianten jeweils nur eine sprachliche Form richtig sein kann (vgl. Milroy 2001: 535). Klein (2018: 32) 
spricht in diesem Zusammenhang von einer „Richtig-Falsch-Ideologie“, die sich mit der Ideologie der Standardsprache zu „einer festen Burg der sprachlichen Normativität“" verbindet.

Hier gibt es in den untersuchten Foren durchaus verschiedene Positionen. Sie reichen von der Weigerung, „eine private Schreibweisenvorstellung außerhalb des für A grundlegend akzeptieren Sprachgebrauches nach dem ÖWB [= Österreichisches Wörterbuch, Anm. HAW] überhaupt lesen zu wollen“ (P-696), bis hin zur Feststellung, dass „Normen (nämlich grammatikalische!) [...] veränderbar" seien (P-436).

Diese zwei Positionen stehen sich unvereinbar gegenüber, da sie sehr unterschiedliche Weltbilder vertreten. Auf der einen Seite wird Sprache als natürlich gesehen, auf der anderen Seite als sozial konstruiert, wie dieser Austausch zwischen drei Personen zeigt:

\footnotetext{
VDS-0136: Entweder oder

Die DEUTSCHE Sprache hat Regeln.

Die DEUTSCHE Sprache basiert nicht auf weiblichen Hormonschüben

VDS-0137: Diese Regeln wurden aber (hauptsächlich) von männlichen Hormonschübern gemacht. Abgesehen davon hat jede Sprache ihre diachronische und synchronische Dimension. Bildung hilft!

VDS-0138: [...] Nein, sondern sie hat sich im Laufe der Zeit entwickelt, ursprünglich gab es im Deutschen sogar überhaupt keine Unterscheidung in Femininum und Maskulinum [...] $>$ Bildung hilft!

Ja, dann fang endlich einmal an, dir welche anzueignen, z.B. in Grammatik.
}

Dieser Ausschnitt zeigt, dass sich ein Standpunkt, der auf der Existenz von Regeln, die sich natürlich entwickelt haben, und ein Standpunkt, der auf der sozialen Konstruiertheit von sprachlichen Regeln basiert, nicht in einem Austausch von Kurztexten konsolidieren lassen. Außerdem wird hier noch einmal die Wichtigkeit von „Bildung“ als implizite Voraussetzung dafür sichtbar, an den Teilen der Debatte teilnehmen zu dürfen, die sich mit linguistischem Wissen beschäftigen.

In den Ausgangstexten wird die Genus-Sexus-Debatte mit „Generalirrtum“ (Maron et al. 2019: o. S.) bezeichnet und es werden die Begriffe grammatisches und natürliches Geschlecht verwendet. Trotzdem kommt der Begriff Genus in den Foren 50-mal vor, proportional zu der Größe des Forums gleich oft im VDSForum wie im Posch-Forum. Bei 28 Stellen lässt sich das Genus des Begriffs nicht im Satz erkennen, meist wird Genus ohne Artikel verwendet. Interessanterweise wird Genus in den übrigen Fällen 15-mal als männliches Nomen behandelt, doppelt so oft wie als neutrales. Dabei lässt sich beobachten, dass der Begriff in Threads von zwei Personen oft von beiden verwendet wird, sobald 
eine Person ihn einführt. Das könnte bedeuten, dass der Begriff nicht für alle, die ihn verwenden, zu ihrem ursprünglichen Wortschatz gehört und das mag auch die Unsicherheit beim korrekten Genus von Genus erklären. Es zeigt sich hier also ein Bedarf, bei Fachvokabular nicht zurückzustehen.

Der Ausdruck generisches Maskulinum wird im Text von Posch verwendet und kommt in etwa gleich oft wie Genus in den Kommentaren vor. Hier werden sowohl bei den Befürworter*innen als auch bei den Gegner*innen meist Aussagen mit Autorentilgung verwendet: „Denn das Generische Maskulinum ist per definitionem geschlechtsneutral, d. h. es spricht weder Männer noch Frauen direkt an und kann damit auch niemanden zusätzlich ,mitmeinen“.” (VDS-0204) oder

Das generische Maskulinum (für alle natürlichen Geschlechter gültig, weil die Tätigkeit hervorhebend und nicht das Geschlecht) ist zwar als eine Möglichkeit im Sprachsystem angelegt, in der Sprachverwendung führt es sich allerdings permanent selbst ad absurdum, weil maskuline Personenbezeichnungen meist als Bezeichnung für die männliche Variante der Person verstanden werden. Das haben einschlägige Experimente immer wieder gezeigt.

(VDS-0225)

Durch Autorentilgung werden Aussagen im Text als Tatsachen dargestellt (vgl. Bendel Larcher 2015: 61). Da sie, anders als im wissenschaftlichen Diskurs, nicht belegt werden, kann das Zustandekommen der Aussage nicht kritisch diskutiert werden. Es kommt, soweit es aus den Kommentaren ersichtlich ist, zu keiner Änderung des eigenen Standpunkts, sondern die Positionen verhärten sich: „Ihr posting widerlegt meine Erklärung nicht, dazu müssen Sie schon auf mein Argument eingehen und nicht nur Ihr altes Argument von oben wiederholen....“ (VDS-0230). Da im Rahmen eines Online-Forums nur verkürzt Positionen dargestellt werden, werden schließlich alle Argumente, auch wissenschaftlich fundierte, zu Meinungen. Das zeigt ein Kommentar in Reaktion auf den Ausgangstext von Posch, in dem Gegenargumente als von Luise F. Pusch bereits vor Jahrzehnten widerlegt beschrieben werden: „[...] keine Autorin hat objektiv widerlegt. Sie hat bloß eine Meinung abgegeben. Und genau dasselbe dürfen auch Gegner der [sic] Genderns machen.“(P-032)

Aber auch Kommentare, die nicht deagentiviert sind und direkt auf den wissenschaftlichen Diskurs verweisen, wie „[a]nders als Grimm, geht K. Brugmann davon aus, dass Sexus Genus parasitär verwendet, und Genus eigentlich die Kategorie Numerus realisiert [...]“ (VDS-0640) ziehen keine differenziertere Diskussion nach sich, sondern lösen wiederum kaum Reaktionen aus. Hier zeigt sich wieder, dass ein zu starker Fachkontext wenig Interaktion hervorruft. 
Es wird neben dem Begriff generisches Maskulinum auch der Begriff generisches Femininum für die Wörter Geisel oder Person verwendet, und das Verschweigen des generischen Femininums als Indiz für eine unaufrichtige Diskussionskultur gesehen.

Generisches Maskulinum ist *keine` männliche Form. Es ist ... ein generisches Maskulinum.

Es gibt auch ein generisches Femininum welches *keine ${ }^{\star}$ weibliche Form ist. Aber das kehren wir in dieser Diskussion immer gerne unter den Tisch weil es sonst ganz einfach keinen Grund für eine Diskussion gäbe.

(VDS-1375)

Das sogenannte generische Femininum ist bereits in einem 2014 in Österreich publizierten offenen Brief gegen geschlechtergerechte Sprache im Rahmen der sogenannten Ö-NORM-Debatte 2014 ein Thema, als ein Vorschlag des Austrian Standards Institutes, das Generische Maskulinum als Norm festzulegen, eine breite mediale Debatte auslöste. Mairhofer \& Posch (2017: 48) nehmen diesen Aspekt in ihrer Analyse des Briefes auf und erklären, dass Wörter mit femininem Genus, die nicht moviert werden können und daher nicht Teil eines Oppositionspaares sind, nicht als generisch bezeichnet werden können. In weniger als der Hälfte der Fälle wird in den Kommentaren das generische Femininum als das bezeichnet, was es in der Fachdiskussion beschreibt, nämlich das Verwenden weiblicher Formen als geschlechtsabstrahierend, wie es mit viel medialer Beachtung an der Universität Leipzig vor einigen Jahren (vgl. Stefanowitsch 2013) oder in Österreich von der Grünen Partei in Kärnten (vgl. Die Grünen Kärnten 2016) eingeführt wurde. Wo die abweichende Definition des generischen Femininums ihren Ursprung genommen hat und wie weit sie verbreitet ist, lässt sich anhand der vorliegenden Daten nicht klären. Die Symmetrie, die dadurch vermeintlich im Sprachsystem hergestellt wird, unterstützt die Position, dass das Generische Maskulinum nicht diskriminierend sei. Die ursprüngliche Idee des generischen Femininums als „einfach alles in der weiblichen Form schreiben“ (VDS-0428) wird in den Foren häufig vorgeschlagen und diskutiert, allerdings nur selten mit dem Fachbegriff bezeichnet.

Ein Punkt, an dem stark mit einer semantischen Verschiebung argumentiert wird, ist die Neutralisierung durch Präsenspartizipien wie Studierende. Sie werden im Aufruf des VDS als Beispiele für „lächerliche Sprachgebilde“ zusammen mit „Luftpiratinnen“ und „Idiotinnen“ erwähnt (Maron et al. 2019), dabei steht jedoch nicht die Betonung des Verlaufs der Verbhandlung (vgl. Kotthoff \& Nübling 2018: 149) im Vordergrund, die die Grundlage für die Kritik in den Foren bildet. Die Frage der Korrektheit - es sei „einfach falsch“ (VDS-0752) - wiegt aber in der Diskussion wesentlich schwerer als die im Ausgangstext angespro- 
chene ästhetische Dimension. Hier werden die Partizipialformen durchgehend auch als solche benannt.

Umgekehrt ist die Frage nach der spezifischen oder generischen Referenz ein Thema, das ohne Fachvokabular geführt wird. Hier wird von „persönliche[r] Anrede“ statt „allgemeine[m] Gendern“ (VDS-1319), von einer „bestimmte[n] Person“ oder einer „allgemeingültige[n] Aussage“ (VDS-1049) gesprochen. Diese Frage der Referenz erscheint in der Diskussion implizit verknüpft mit der Frage nach Singular und Plural, wie es auch in der Fachdiskussion festgestellt wurde (vgl. Kotthoff \& Nübling 2018: 92). Diese Verbindung wird nicht explizit hergestellt, aber das Generische Maskulinum wird als Pluralregel wahrgenommen, als „Regel, dass gemischte Gruppen mit dem Plural des maskulinen Singular [sic] bezeichnet werden“ (VDS-1033). Da diese häufigen Singular-PluralBeobachtungen nicht zusammen mit Bemerkungen zur Referenz auftreten, liegt der Schluss nahe, dass Singular und Plural hier zum Teil stellvertretend für Referenz auftreten und eine Lücke füllen, die durch das Vernachlässigen von Fragen der Referenz in der öffentlichen Diskussion auftritt. Während der Duden-Band zum richtigen Gendern diese Frage behandelt (vgl. Diewald \& Steinhauer 2017: 72-80), wird sie in Zeitungskommentaren und Leitfäden selten aufgegriffen.

\section{Schlussbemerkungen}

Die Analyse hat gezeigt, dass die Linguistik und die Deutsche Philologie in der Debatte ein hohes Ansehen besitzen und sie pauschal zur Bestärkung der eigenen Meinung herangezogen werden, dass aber der aktuelle Stand der Forschung, insbesondere der hier relevanten Psycho- und Kognitionslinguistik, weitgehend unbekannt ist. Der Wunsch, empirische Studien im Original zu diskutieren, scheitert an der Zugänglichkeit der Publikationen, die Lücke füllen Zeitungsartikel, Blogs und Youtube-Videos. Wenn Vertreter*innen der Linguistik der eigenen Meinung explizit widersprechen, wie in den vorliegenden Daten durch den zweiten Zeitungsartikel oder die Referenzen darin, wird diesen Personen eher abgesprochen, die Linguistik repräsentieren zu können, als die Linguistik als Disziplin anders zu bewerten.

Kenntnisse der Linguistik werden zu einem großen Teil mit Kenntnissen der Grammatik gleichgesetzt und die Frage der sprachlichen Korrektheit ist zentral. Es ist mehr Fachvokabular zur Grammatik als zu anderen Bereichen der Linguistik, z. B. der Referenzlinguistik, bekannt. Die Argumentation der grammatikalischen Korrektheit klammert die soziale Komponente von Sprache weitgehend 
aus, stößt so aber auch auf Widerspruch. Anders als in anderen Teilen des Forums wird hier, wo es um Grammatik geht, immer wieder das Kriterium der Bildung als Berechtigung angeführt, an der Diskussion teilnehmen zu dürfen. Der Umstand, dass Fachvokabular nicht immer korrekt verwendet wird, deutet darauf hin, dass das Vorzeigen von Bildung und linguistischem Wissen hier in Hinsicht auf den Vertikalitätsstatus, also die Einnahme eines Expertenstatus, wichtig ist. Trotz der Wichtigkeit von formaler Bildung in dieser Auseinandersetzung beruft sich niemand darauf, aktuell an Universitäten beschäftigt zu sein, denn europäische Universitäten werden als Orte der Ideologie gezeichnet.

Durch den Anspruch auf Moral, der den Vertreter*innen geschlechtergerechter Sprache zugesprochen wird, muss im Zuge der Positionierung das im Sinne von Du Bois (2007) bewertete Objekt, also der geschlechtergerechte Sprachgebrauch, abgewertet werden, um eine moralisch integre Position einzunehmen. Diese Abwertung geschieht hier unter anderem über die Bewertung der geschlechtergerechten Sprache als grammatikalisch falsch und als durch empirische Studien widerlegt, sodass die Diskursteilnehmenden sich selbst als Expert^innen positionieren, die nicht unmoralisch, sondern informierter handeln. Die Frage des Einflusses von Sprache auf die gesellschaftliche Entwicklung wird dabei als Ideologie und als unwissenschaftlich bewertet. Auch Befürworter^innen von geschlechtergerechter Sprache grenzen sich von dem Schreckgespenst der „Genderriege“ (P-204) in den Daten immer wieder dadurch $\mathrm{ab}$, dass sie sich gegen den ausgeübten Zwang stellen, der dieser zugeschrieben wird, bzw. argumentieren, dass es diesen Zwang oder diese Gruppe nicht gebe. Hier lassen sich also zwei Stance-Dreiecke nach Du Bois (2007) zeichnen, die einmal die geschlechtergerechte Sprache als Objekt und einmal den Zwang zur geschlechtergerechten Sprache als Objekt beinhalten. In beiden Fällen erfolgt eine Abgrenzung zur selben Gruppe, die entweder ideologisch verblendet und uninformiert handelt, oder ihre gesellschaftliche Macht missbraucht und diktatorisch handelt. Die Gruppe ist ein Personentypus, aber kein Akteur, der in den Diskussionen selbst auftritt. Wenn der Verdacht besteht, ein Posting stamme von dieser Personengruppe, z. B. über die Zuschreibung „ich als Sprachwissenschafterin“ (VDS-0937), bricht die Kommunikation meist ab. Ob das nur für diese beiden Foren, für den spezifischen Kontext Online-Forum oder für den gesamten Diskurs kennzeichnend ist, kann nur durch die Analyse einer größeren Datenmenge festgestellt werden.

Im Diskurs zur geschlechtergerechten Sprache werden linguistische und gesellschaftliche Themen eng verknüpft und dadurch Sprachideologien deutlich sichtbar. Die Sprache als wertfreies, natürlich gewachsenes gesellschaftliches Gut mit unveränderbaren Regeln steht der Auffassung der Sprache als 
Mittel zur Konstruktion gesellschaftlicher Wirklichkeit gegenüber. Die Linguistik als Hüterin dieses gesellschaftlichen Guts genießt Autorität, aktuelle Entwicklungen, die dem eigenen Standpunkt widersprechen, werden aber nicht wahrgenommen oder als nicht genuin sprachwissenschaftlich bezeichnet. So bleibt das Bild der Sprachwissenschaft unscharf.

Blommaert (1999: 425-428) betont die Unregelmäßigkeit sprachlicher Entwicklung. Historisch gebe es Phasen, in denen sich Sprache nur langsam entwickelt und Phasen, in denen Sprache Ziel politischer, sozialer und kultureller Intervention wird, die oft auch mit Fragen der nationalen Identität verbunden sind. Die in diesem Text nachgezeichnete heftige Diskussion, die für dieses Thema in den letzten Jahren typisch ist, weist auf die aktuelle kulturelle und politische Signifikanz dieser Sprachformen hin, die eine Untersuchung so lohnend erscheinen lässt. Dieser Beitrag konnte nur einen kleinen Teil des Diskurses in Augenschein nehmen. Eine größer angelegte diskurslinguistische Untersuchung, die verschiedene Textsorten über einen längeren Zeitraum sowohl qualitativ als auch quantitativ analysiert, könnte für die Rolle der Linguistik in der Gesellschaft aufschlussreich sein und wesentlich zur Erforschung von aktuellen Sprachideologien beitragen.

\section{Literaturverzeichnis}

\section{Quellen}

Maron, Monika, Wolf Schneider, Walter Krämer \& Josef Kraus (2019): Schluss mit dem GenderUnfug! Ein Aufruf zum Widerstand aus dem Verein Deutsche Sprache. Der Standard. https://www.derstandard.at/story/2000099137126/schluss-mit-dem-gender-unfug (letzter Zugriff 22.02.2020).

Forum VDS = Postings zum Beitrag von Maron et al. (2019): https://www.derstandard.at/story/2000099137126/schluss-mit-dem-gender-unfug (letzter Zugriff 31.07.2019)

VDS-0001 28. März 2019, 13:56:12 bis VDS-1405 8. März 2019, 00:14:35.

Posch, Claudia (2019): Der wahre Sprachunfug - eine Replik. Der Standard. https://www.derstandard.de/story/2000099886356/der-wahre-sprachunfug-eine-replik (letzter Zugriff 22.02.2020).

Forum Posch $=$ Postings zum Beitrag von Posch (2019). https://www.derstandard.de/story/2000099886356/der-wahre-sprachunfug-eine-replik (letzter Zugriff 06.04.2019)

P-001 29. März 2019, 00:21:43 bis P-752 22. März 2019, 04:11:04. 


\section{Literaturnachweise}

Bayer, Klaus (2007): Argument und Argumentation. Logische Grundlagen der Argumentationsanalyse (Studienbücher zur Linguistik). 2. Aufl. Göttingen: Vandenhoeck \& Ruprecht.

Bendel Larcher, Sylvia (2015): Linguistische Diskursanalyse. Ein Lehr- und Arbeitsbuch (Narr Studienbücher). Tübingen: Narr Francke Attempo.

Blommaert, Jan (1999): The debate is closed. In Jan Blommaert (Hrsg.), Language Ideological Debates (Language, Power and Social Process 2), 425-438. Berlin, New York: Mouton de Gruyter.

Braun, Friederike, Anja Gottburgsen, Sabine Sczesny \& Dagmar Stahlberg (1998): Können Geophysiker Frauen sein? Generische Personenbezeichnungen im Deutschen. Zeitschrift für Germanistische Linguistik 26 (3), 265-283.

Bundesministerium für Wissenschaft, Forschung und Wirtschaft (BMWFW) (2017): Bürgerinitiative Nr. 114 betr. "Wissenschaftliche Arbeiten genderfrei!", Beantwortung. 260/SBI vom 19.07.2017 zu 114/BI (XXV.GP). https://www.parlament.gv.at/PAKT/VHG/XXV/SBI/SBI_00260/imfname_649838.pdf (letzter Zugriff 22.02.2020).

Busch, Brigitta (2019): Sprachreflexion und Diskurs: Theorien und Methoden der Sprachideologieforschung. In Gerd Antos, Thomas Niehr \& Jürgen Spitzmüller (Hrsg.), Handbuch Sprache im Urteil der Öffentlichkeit (Handbücher Sprachwissen 10), 107-139. Berlin, Boston: De Gruyter.

Die Grünen Kärnten (2016): Grüne Klagenfurt: Die Frauen vor den Vorhang holen! Presseaussendung. https://www.ots.at/presseaussendung/OTS_20160210_OTS0161/grueneklagenfurt-die-frauen-vor-den-vorhang-holen (letzter Zugriff 22.02.2020).

Dietz, Hella (2018): Jordan Peterson: Mythos Tabubrecher. Die Zeit, 10 nach 8. https://www.zeit.de/kultur/2018-08/jordan-peterson-kanada-professor-politischekorrektheit-feminismus-geschlechteridentitaet/komplettansicht (letzter Zugriff 22.02.2020).

Diewald, Gabriele \& Anja Steinhauer (2017): Richtig gendern. Wie Sie angemessen und verständlich schreiben. Berlin: Dudenverlag.

Doleschal, Ursula (2002): Das generische Maskulinum im Deutschen. Ein historischer Spaziergang durch die deutsche Grammatikschreibung von der Renaissance bis zur Postmoderne. Linguistik Online 11 (2), 39-70.

Du Bois, John W. (2007): The stance triangle. In Robert Englebretson (Hrsg.), Stancetaking in Discourse: Subjectivity, Evaluation, Interaction (Pragmatics \& Beyond New Series 164), 139-182. Amsterdam, Philadelphia: John Benjamins Publishing Company.

Gardt, Andreas (2007): Diskursanalyse - Aktueller theoretischer Ort und methodische Möglichkeiten. In Ingo H. Warnke (Hrsg.), Diskurslinguistik nach Foucault. Theorie und Gegenstände (Linguistik, Impulse \& Tendenzen 25), 27-52. Berlin, New York: De Gruyter.

Gygax, Pascal, Ute Gabriel, Oriane Sarrasin, Jane Oakhill \& Alan Garnham (2008): Generically intended, but specifically interpreted: When beauticians, musicians and mechanics are all men. Language and Cognitive Processes 23, 464-485.

Huster, Alina: (2018): Das war das Community-Jahr 2017. Der Standard. https://www.derstandard.at/story/2000070294686/das-war-das-community-jahr-2017 (letzter Zugriff 22.02.2020). 
Irmen, Lisa \& Ute Linner (2005): Die Repräsentation generisch maskuliner Personenbezeichnungen. Zeitschrift für Psychologie / Journal of Psychology 213 (3), 167-175.

Irmen, Lisa \& Vera Steiger (2005): Zur Geschichte des Generischen Maskulinums: Sprachwissenschaftliche, sprachphilosophische und psychologische Aspekte im historischen Diskurs / On the history of the generic use of the masculine gender: Linguistic, philosophical, and psychological aspects in historical discourse. Zeitschrift für germanistische Linguistik 33 (2-3), 212-235.

Kalverkämper, Hartwig (1979): Die Frauen und die Sprache. Linguistische Berichte 62, 55-71. Kelle, Birgit \& Rohleder, Franz (2015): Bestseller-Autorin Birgit Kelle: So irre ist der GenderWahn. In Merkur. https://www.merkur.de/politik/bestseller-autorin-birgit-kelle-irregender-wahn-gender-gaga-4790683.html (letzter Zugriff 22.02.2020).

Kienpointner, Manfred (1992): Alltagslogik. Stuttgart, Bad Cannstatt: Frommann-Holzboog. Klein, Wolf Peter (2018): Sprachliche Zweifelsfälle im Deutschen: Theorie, Praxis, Geschichte (De Gruyter Studium). Berlin, Boston: De Gruyter.

Kotthoff, Helga \& Damaris Nübling (2018): Genderlinguistik. Eine Einführung in Sprache, Gespräch und Geschlecht (Narr Studienbücher). Tübingen: Narr Francke Attempto.

Kusterle, Karin (2011): Die Macht von Sprachformen. Der Zusammenhang von Sprache, Denken und Genderwahrnehmung. Frankfurt a. M.: Brandes \& Apsel.

Leiss, Elisabeth (1994): Genus und Sexus. Kritische Anmerkungen zur Sexualisierung von Grammatik. Linguistische Berichte 152, 281-300.

Mairhofer Elisabeth \& Claudia Posch (2017): Die Normalität ist eine gepflasterte Straße ... Argumentationsanalytische Untersuchung eines Offenen Briefs gegen antidiskriminierenden Sprachgebrauch in Österreich. Osnabrücker Beiträge zur Sprachtheorie 91, 35-57.

Milroy, James (2001): Language ideologies and the consequences of standardization. Journal of Sociolinguistics 5 (4), 530-555.

Pérez, Efrén 0. \& Margit Tavits (2019): Language Influences Public Attitudes toward Gender Equality. The Journal of Politics 81 (1), 81-93.

Peterson, Jordan (2019): Professor Against Political Correctness, Youtube Playlist. https://www.youtube.com/playlist?list=PL22J3VaeABQD8oW-mqWpKumeqglQCe6VZ (letzter Zugriff 22.02.2020).

Pettersson, Magnus (2011): Geschlechtsübergreifende Personenbezeichnungen. Eine Referenzund Relevanzanalyse an Texten (Europäische Studien zur Textlinguistik 11). Tübingen: Narr Francke Attempo.

Pusch, Luise F. (1979): Der Mensch ist ein Gewohnheitstier, doch weiter kommt man ohne ihr Eine Antwort auf Kalverkämpers Kritik an Trömel-Plötz’ Artikel über „Linguistik und Frauensprache“. Linguistische Berichte 63, 84-102.

Ring Freiheitlicher Studenten (RFS) (2017): Wissenschaftliche Arbeiten genderfrei! Presseaussendung. https://www.ots.at/presseaussendung/OTS_20170427_OTS0038/wissenschaftlichearbeiten-genderfrei-bild (letzter Zugriff 22.02.2020).

Ring Freiheitlicher Studenten (RFS) (o. J.): Programm. Website. https://www.rfs.at/programm/ (letzter Zugriff 22.02.2020).

Scholten, Daniel (2018): Das Genus ist dem Sexus sein Nexus. Die empirische Forschung der feministischen Linguistik. Video, Website Belles Lettres. https://www.belleslettres.eu/content/deklination/gender-nubling-lobinsueddeutsche.php (letzter Zugriff 22.02.2020). 
Spitzmüller, Jürgen (2013): Metapragmatik, Indexikalität, soziale Registrierung. Zur diskursiven Konstruktion sprachideologischer Positionen. Zeitschrift für Diskursforschung 3, 263-287.

Spitzmüller, Jürgen (2019): ,Sprache‘ - ,Metasprache‘ - ,Metapragmatik‘: Sprache und sprachliches Handeln als Gegenstand sozialer Reflexion. In Gerd Antos, Thomas Niehr \& Jürgen Spitzmüller (Hrsg.), Handbuch Sprache im Urteil der Öffentlichkeit (Handbücher Sprachwissen 10), 11-30. Berlin, Boston: De Gruyter.

Spitzmüller, Jürgen \& Ingo H. Warnke (2011): Diskurslinguistik. Eine Einführung in Theorien und Methoden der transtextuellen Sprachanalyse (De Gruyter Studium). Berlin, Boston: De Gruyter.

Spitzmüller Jürgen, Mi-Cha Flubacher \& Christian Bendl (2017): Soziale Positionierung: Praxis und Praktik. Einführung in das Themenheft. Wiener Linguistische Gazette 81, 1-18.

Stefanowitsch, Anatol (2013): Das generische Femininum und die Gegner des Femininums. Website Sprachlog. http://www.sprachlog.de/2013/10/14/das-generische-femininumund-die-gegner-des-femininums/ (letzter Zugriff 22.02.2020).

Stefanowitsch, Anatol (2018): Eine Frage der Moral. Warum wir politisch korrekte Sprache brauchen. Berlin: Dudenverlag.

Teireck, Jana (2019): Sprache und Diskriminierung: Soziale Ungleichheit als Gegenstand emanzipatorischer Sprachpolitik. In Gerd Antos, Thomas Niehr \& Jürgen Spitzmüller (Hrsg.), Handbuch Sprache im Urteil der Öffentlichkeit (Handbücher Sprachwissen 10), 383-399. Berlin, Boston: De Gruyter.

Trömel-Plötz, Senta (1978): Linguistik und Frauensprache. Linguistische Berichte 57, 49-68.

Volksanwaltschaft (2018): Bürgerinitiative Nr.30/BI-NR/2017, Stellungnahme der Volksanwaltschaft. https://www.volksanwaltschaft.gv.at/downloads/ad917/Stellungnahme\%20VAAusschuss $\% 20$ \%C3\%BCr\%20Petitionen\%20und\%20B\%C3\%BCrgerinitiativen_A1017Wien.pdf (letzter Zugriff 22.02.2020).

Wetschanow, Karin (2017): Von nicht-sexistischem Sprachgebrauch zu fairen W_ortungen - Ein Streifzug durch die Welt der Leitfäden zu sprachlicher Gleichbehandlung. Osnabrücker Beiträge zur Sprachtheorie 90, 33-59. 